\title{
Perineal Groove: A Rare Congenital Midline Defect of Perineum
}

\author{
Mimily Harsono, MD, MSc ${ }^{1}$ Massroor Pourcyrous, $\mathrm{MD}^{1,2,3}$ \\ 1 Division of Neonatal-Perinatal Medicine, Department of Pediatrics, \\ University of Tennessee Health Science Center, Memphis, Tennessee \\ 2 Department of Obstetrics/Gynecology, University of Tennessee \\ Address for correspondence Mimily Harsono, MD, MSc, Division of \\ Neonatal-Perinatal Medicine, Department of Pediatrics, 853 Jefferson \\ Health Science Center, Memphis, Tennessee \\ Avenue, Suite-201, Memphis, TN 38103 (e-mail: mharsono@uthsc.edu) \\ ${ }^{3}$ Department of Physiology, University of Tennessee Health Science \\ Center, Memphis, Tennessee \\ Am J Perinatol Rep 2016;6:e30-e32.
}

\begin{abstract}
Keywords

- congenital anomaly

- perineal groove

- perineal defect

- newborn

Perineal groove is a rare congenital malformation that is characterized by an exposed wet sulcus with nonkeratinized mucous membrane that extends from the posterior vaginal fourchette to the anterior ridge of the anal orifice. This condition is one of the uncommon anomalies of urogenital/anogenital region that is unknown to many clinicians. Although, this condition may be self-resolved before the age of 2 years, this nonepithelized mucous membrane can pose the risk of local irritation and infection, urinary tract infection, and the possibility of nonself-resolved condition that eventually needs surgical correction. Only a few reported cases $(n=23)$ were found in current medical literatures. This lesion could be misdiagnosed as contact dermatitis, trauma, or even sexual abuse. Therefore, recognition of the congenital perineal groove at birth is important for the health care providers to deliver an appropriate parental counseling and appropriate follow-up.
\end{abstract}

Congenital perineal groove is characterized by an exposed erythematous nonepithelized mucous membranes that resembles exposed wet erythematous sulcus, inflammation, or rupture of the midperineum area. This lesion extends vertically downward from the posterior portion of the vaginal fourchette to the anterior rim of the anus. ${ }^{1-3}$ This lesion resembles failure of mid-perineum fusion or failure of midperineum skin epithelialization. ${ }^{3}$

\section{Case Presentation and Management}

\section{Case 1}

At 37 weeks gestational age (GA) a white female with birthweight (BW) of 3,450 $\mathrm{g}$ was delivered by cesarean section (CS) due to recurrent CS and placenta previa. Apgar scores were 8 and 9 at 1 and 5 minutes, respectively. Infant was admitted to the neonatal intensive care unit (NICU) for suspected imperforated anus. Infant's mother was a 25 -year-old gravida 3 and para 3 (G3P3) with good prenatal care. All prenatal tests were negative and there was no history of smoking, drinking alcohol, or abusing substances. Placental histopathology showed mild placentomegaly, acute chorioamnionitis without funisitis, and placenta previa. There was no family history of congenital anomalies.

Physical examination of the infant was normal except for the perineal area that showed a lesion, which was suspicious for congenital or iatrogenic perineal rupture or imperforated anus. The perineal defect was noted stretching vertically upward from 12 o'clock position of the anal rim toward the posterior vaginal fourchette, with a wet groove-like unkeratinized erythematous mucosal lesion (-Fig. 1). Urethra and vaginal orifices were intact and within appropriate position. Infant did not show any signs of urination problem. The diagnosis of imperforated anus was ruled-out with normal anorectal examination and infant was stooling from the wellpositioned anal opening located posterior to the lesion. The received

April 18, 2015

accepted after revision

August 20, 2015

published online

October 28, 2015
DOI http://dx.doi.org/

$10.1055 / \mathrm{s}-0035-1566311$. ISSN 2157-6998.
Copyright (c) 2016 by Thieme Medical

Publishers, Inc., 333 Seventh Avenue, New York, NY 10001, USA. Tel: +1(212) 584-4662.
License terms

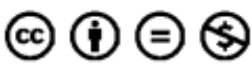




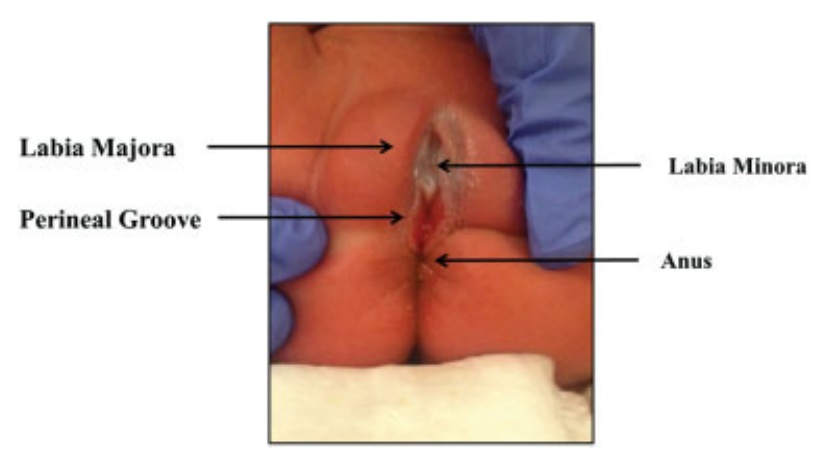

Fig. 1 (Case 1) Wet groove between the vulva and anus.

ultrasounds of the head, pelvic, renal, and spine were normal. The skeletal survey was also normal, and an echocardiogram showed a small secundum atrial septal defect with a tiny patent ductus arteriosus. The hospital course was uneventful except for transient hypoglycemia and received empiric antibiotics for suspected infection with negative blood culture. She was discharged home at 5 days of life. Follow-up at 4 months of age revealed no complications.

\section{Case 2}

At 37 weeks GA an African American female with BW of 3,510 g was delivered by CS due to recurrent CS and preeclampsia. Apgar scores were 7 and 8 at 1 and 5 minutes, respectively. Pregnancy was complicated by insulin-controlled gestational diabetes, chronic hypertension, and preeclampsia. Infant was admitted to the NICU for transient hypoglycemia and suspected perforated perineum with a four-vessel umbilical cord (two veins and two arteries). Mother was a 41-year-old G5P5 with good prenatal care. All prenatal tests were negative and there was no history of drinking alcohol, smoking, or abusing substances. Placental histopathology was normal except for increased fetal nucleated red blood cells consistent with fetal hypoxia. There was no family history of congenital malformations.

Infant's physical examination was normal for all organ systems except for four-vessel umbilical cord and perineal defect. The perineal defect was a wet groove-like erythematous nonepithelized mucous membrane that extended vertically downward from 6 o'clock position of the posterior vaginal fourchette to the anterior rim of the anal orifice and resembled ruptured perineal area (-Fig. 2). Urethra, vaginal, and anal orifices were intact and well-positioned. Stooling originated from an intact anal opening, and no fecal or urinary incontinence was noted. Head, renal, pelvic, and spine ultrasounds were all unremarkable. Echocardiogram to rule out any congenital cardiac anomaly that could be associated with fourvessel cord was reported as normal. Skeletal survey was normal. Hospital course was unremarkable and she was discharged home with her parents at 3 days of life. At 1-year follow-up, it showed healing of the perineal groove lesion.

\section{Discussion}

Congenital perineal groove is a rare and benign malformation. It is mostly presented in female infants as an isolated anoma-

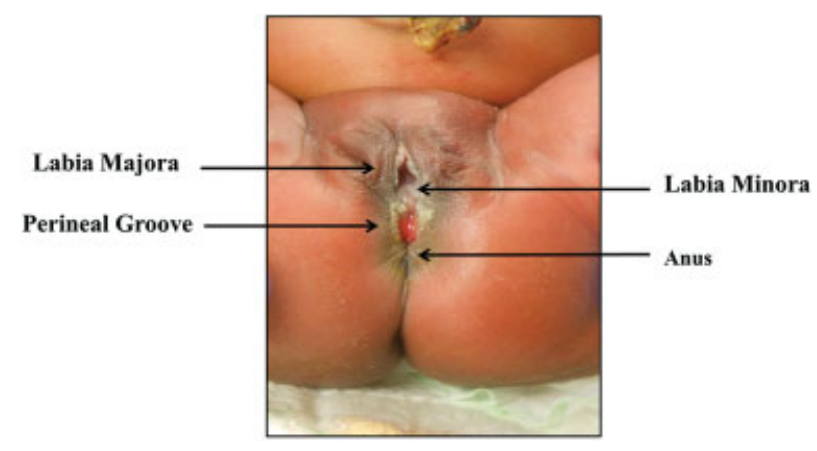

Fig. 2 (Case 2) Wet groove between the vulva and anus.

ly. So far only 23 cases have been reported in medical literatures. ${ }^{1-11}$ In rare cases, it may be associated with other regional anomalies of anogenital and/or urogenital system such as anteriorly placed anus, ectopic anus, prolapsed anus, or urinary tract malformation. ${ }^{3,6,12}$ Both of our patients were delivered within 12-month period and were diagnosed at birth. It is likely that the incidence of this congenital anomaly is underestimated as it may be unrecognized earlier at birth, and/or later misdiagnosed as a diaper rash, contact dermatitis, or trauma. ${ }^{5,6}$

In 1968, Stephens ${ }^{3}$ described perineal groove as a congenital malformation that consists of three features: (1) a wet groove in midperineum between the fourchette and the anus; (2) normal formation of the vestibule including the urethra and vagina; and (3) hypertrophy of the minoral tails that skirt the perineum and course posteriorly to join at the anus or to surround it.

Pathogenesis of perineal groove remains unclear. However, embryological mechanisms have been proposed as follows: (1) a relic of the open cloacal duct, ${ }^{13}$ (2) midline fusion failure of medial genital folds between the perineal raphe and the vestibule, ${ }^{3,4}$ and (3) urorectal septum developmental defect during cloacal embryological stages at 5 th to 8 th week of GA. ${ }^{6,7,14}$ Given that the perineal groove anomaly was also reported in one male infant, ${ }^{12}$ this congenital anomaly can be derived during the embryological development stage of external genitalia. External genitalia of both sexes are developed from the genital tubercle, genital folds, and labioscrotal folds. Therefore, failure of labioscrotal folds fusion to form perineal raphe will cause perineal groove to occur. ${ }^{2}$

Histological reviews of the resected area varied from a nonkeratinized stratified squamous epithelium without sebaceous glands or sweat glands or hair follicles, ${ }^{3,4,15}$ to a simple columnar or stratified columnar or cuboidal epithelium of a rectal type mucosa with intervening area of a nonkeratinized stratified squamous epithelium. ${ }^{7,15}$ These findings resembled anorectal transitional zone epithelium, which implied that this malformation probably associated with embryology defect during urorectal septum development. ${ }^{2,6,7}$

Patients with this congenital malformation are at an increased risk of urinary tract infection, inflammation, or infection of the nonepithelized mucosa, fecal continence, or incontinence and other associated regional anomaly. ${ }^{5,6}$ This lesion may be self-resolved and completely epithelized by the age of 1 to 2 years. ${ }^{1,2,5,6}$ Surgical correction can be done 
mainly for cosmetic reason, if the lesion is not epithelialized by the age of 2 years. However, there are also some reports that the lesion may need surgical correction earlier than the age of 2 years for clinical reasons such as repeated inflammation or infection of the nonepithelized area from exposure to mucous drainage, wet secretion from the urethra, vagina, or anal sites. ${ }^{5,6}$ Because this lesion resembles rupture or inflammation of perineum region, failure to recognize this lesion at birth will lead to misdiagnosis such as infection, irritant dermatitis, ulcerated hemangioma, lichen sclerosis, trauma, or even sexual abuse. ${ }^{5}$

\section{Conclusion}

This uncommon benign congenital malformation tends to be self-epithelialized. However, surgical referral is warranted if it involves other regional anomalies, recurrent infection, or ulceration of the perineal groove, failure of epithelialization after the age of 2 years or for a cosmetic reason. Early recognition of congenital perineal groove at birth is important for health care providers to deliver an informed parental counseling, appropriate follow-up, and to prevent an unnecessary surgical or medical intervention.

\section{Funding Source}

No external funding.

\section{Financial Disclosure}

The authors do not have any financial relationships relevant to this article to disclose.

Conflict of Interest

The authors do not have any potential conflict of interest to disclose.

\section{Contributors' Statement}

Dr. Harsono was involved in diagnosing the malformation, the literature review, obtaining the institutional review board approval, acquisition of information, writing the first draft, and the revision of the case report. Dr. Pourcyrous was involved in diagnosing the malformation, literature review, critical review, revision, and approval of the final article as submitted.

\section{Permission for Photographs}

All photographs provided in this case report have consent forms signed by patients' parents or the legal guardians for the purpose of medical literature publication and education. This case report which included the photographs has received an Institutional Board Review approval from the University of Tennessee Health Science Center and the Regional One Health Hospital to conduct the study.

\section{References}

1 Kadowaki H, Nakahira M, Yamada C, Takeuchi S, Tamate S, Shiokawa C. Perineal groove and perineal canal. Jpn J Surg 1983; 13(3):216-218

2 Sekaran P, Shawis R. Perineal groove: A rare congenital abnormality of failure of fusion of the perineal raphe and discussion of its embryological origin. Clin Anat 2009;22(7):823-825

3 Stephens FD. The female anus, perineum and vestibule. Embryogenesis and deformities. Aust N Z J Obstet Gynaecol 1968;8(2): 55-73

4 Abdel Aleem A, el Sheikh S, Mokhtar A, Ghafouri H, Saleem M. The perineal groove and canal in males and females-a third look. Z Kinderchir 1985;40(5):303-307

5 Diaz L, Levy ML, Kalajian A, Metry D. Perineal groove: a report of 2 cases. JAMA Dermatol 2014;150(1):101-102

6 Esposito C, Giurin I, Savanelli A, Alicchio F, Settimi A. Current trends in the management of pediatric patients with perineal groove. J Pediatr Adolesc Gynecol 2011;24(5):263-265

7 Mullassery D, Turnock R, Kokai G. Perineal groove. J Pediatr Surg 2006;41(3):e41-e43

8 Siruguppa K, Tuli SS, Kelly MN, Tuli SY. Newborn female with a midline perineal defect. Clin Pediatr (Phila) 2012;51(2):188-190

9 Verma SB, Wollina U. Perineal groove-a case report. Pediatr Dermatol 2010;27(6):626-627

10 Wester T. Perineal groove-an undiagnosed malformation? [in Swedish]. Lakartidningen 2004;101(35):2646-2647

11 Wieringa NM, van der Putten ME. A neonate with an abnormal perineum [in Dutch]. Ned Tijdschr Geneeskd 2011;155(36): A2096

12 Chatterjee SK, Chatterjee US, Chatterjee U. Perineal groove with penoscrotal hypospadias. Pediatr Surg Int 2003;19(7): 554-556

13 van der Putte SC. Normal and abnormal development of the anorectum. J Pediatr Surg 1986;21(5):434-440

14 Stephens FD. Embryology of the cloaca and embryogenesis of anorectal malformations. Birth Defects Orig Artic Ser 1988;24(4): 177-209

15 Bass LM, Wershil BK. Anatomy, histology, embryology and developmental anomalies of the small and large intestine. In: Feldman M, Friedman LS, Brandt LJ eds. Sleisenger and Fordtran's gastrointestinal and liver disease. 10th ed. Philadelphia, PA: Saunders, Elsevier Inc; 2016:1649-1678 